straightened. Subsequently I did some sword exercise (which is like single-stick) and never experienced the slightest difficulty in making the cuts and parries, but my lunge (as with a foil) was a little short. Every summer, dating from two years after the accident, I have done regular sculling on the Thames ; in fact, in 1880 (I think) I twice took a skiff up from Richmond to Cricklade and back, doing about 15 miles a day. If I scull hard I get a kind of numbness in my little finger and part of my third finger ; I suppose the ulnar nerve is slightly compressed in cicatricial tissue at the elbow. I have played billiards just as well after as before the accidents. If I write for a long time I get the numbness above referred to. My pistol-shooting is just as good as ever it was." The figures have been made from skiagraphs of the arm in flexion and in extension. The wide separation of the fragments is noteworthy, also the fibrous union between. As the skiagraphs were taken about 30 years since the accident it is extremely interesting to see that the shaft of the ulna has attempted to grow a small and new olecranon process. Other adaptive changes, lippings of osteo-arthritis, can be seen in the joint, such as enlargements of the anterior lips of the sigmoid cavity of the uIna and the head of the radius. No operation was undertaken in this case and in spite of all that you see the man has a most excellent arm.

The next two cases (Figs. $6 \mathrm{~A}$ and $6 \mathrm{~B}$ and Fig. 7) show the effect of using a screw instead of a wire. The first patient, under the care of Dr. A. G. Mackenzie of Addlestone, broke his olecranon. The olecranon had been united to the shaft of the ulna by means of a strong silk, the knot fastening which had been undone when movements were begun. A little later the silk was removed without any knot on it being present. It was then determined to fasten the detached olecranon process to the shaft of the ulna with a long screw, after freeing it from the humerus. This was done, the fragments being brought together excellently, as shown by the slide from the tracing of a skiagraph that was too dense to permit of a lantern slide being made. A skiagraph was taken a few days after operation and another skiagraph was taken about two months after operation. They show three points of interest; firstly, the screw is loosened, the process appearing to begin round the tip of the screw, where the surrounding bone had become more translucent to the $x$ rays; secondly, the head of the

FIG. 7.

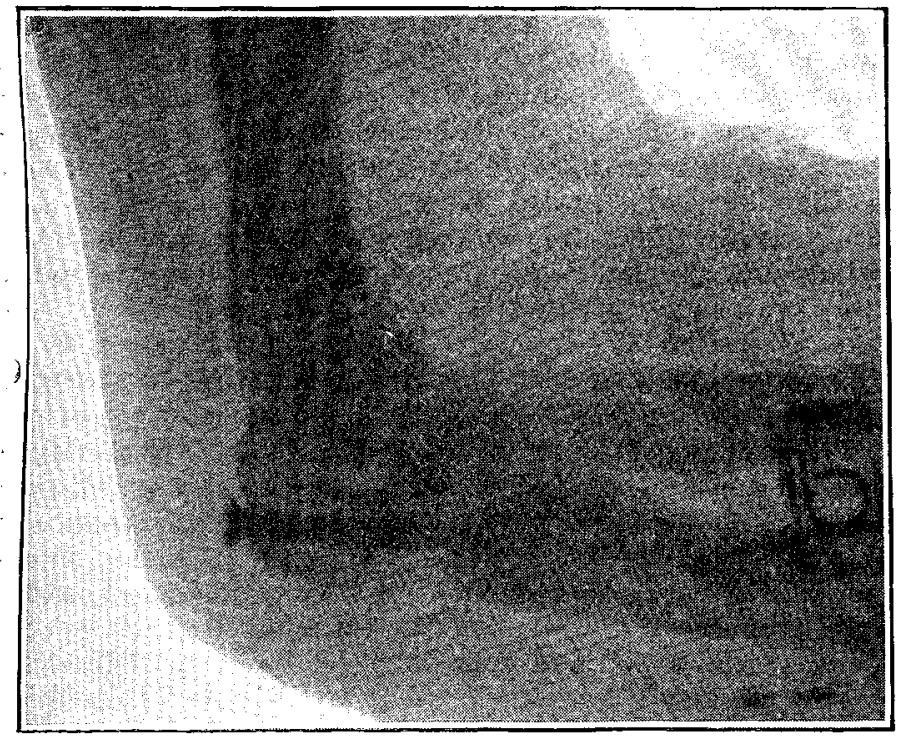

Skiagraph of another case showing a condition similar to that in the tracing $6 \mathrm{I}$, and also the separation of the fragments with the extrusion of the serew.

screw projects appreciably beyond the upper surface of the olecranon with which it was flush in the skiagraph taken shortly after operation; thirdly, the loosening of the screw has allowed the fragments to come apart, which is not shown by the artist in the tracing taken from a bad skiagraph, but easily demonstrated clinically as a gap between the fragments. Tet in spite of this the man has excellent movements and a most useful arm ; full flexion and full extension of the elbow are limited by the fact that in spite of all our precautions the movements of the olecranon on the humerus are impeded by cicatricial contraction of the tissues which join the two together. At a later date the screw, which had become quite loose, was removed without any anæsthetic.

This slide (Fig. 7) is produced from a decent skiagraph of another olecranon which was screwed, the final result being excellent. It shows all the structures as they are and is therefore much better than the previous tracings. It was taken about a month or six weeks after the operation and shows that the fragments have come a little apart from each other and that the head of the screw projects from the olecranon. From this it is quite obvious that it is the fibrous union between the fragments which holds them together and not the loose screw. Internal splints do their work within a given time, like external splints, after which date they can be removed.

The preceding studies of the results of wiring the olecranon have demonstrated the following points. 1. That internal splints lead to absorption of the hard substances of the bone round them. 2. This condition in its turn leads to the loosening of the internal splint and therefore the more easily to its breaking with a sudden effort, to the commencing extrusion of a screw, and to some separation of the fragments. 3. If the part is kept at rest by external splints, in addition the softened bone around the internal splint will harden and re-ossify, bringing about bony union between the fragments. 4. If the part is exercised, as it must be when the fracture is in the neighbourhood of a joint, the fragments are still further separated from each other and the union between them is fibrous, not bony. 5. A perfectly good and excellently functional limb is obtained with such a fibrous union, with some possible limitation of extreme flexion and extreme extension. 6. This limitation is caused by the adhesion or cicatricial contraction of the attachments of the olecranon to the humerus ; putting the triceps at a mechanical disadvantage, arising and being inserted on the same bone. 7. The advantage of direct and internal splintage lies solely in its allowing movements to be begun early, preventing much limitation to the movements of the olecranon on the humerus. 8. From the point of view of the subsequent value of the limb it makes little or no difference whether the union between the fragments is fibrous or bony provided that the olecranon moves freely on the humerus. 9 . It is a matter of indifference as to the nature of the internal splint used, such as silver wire, copper wire, iron wire, or silk. Screws are not so good as sutures in this situation. An absorbable suture is not a good internal splint, because its presence will be required for some weeks at least. 10. For success the operation area must not suppurate. 11. In neglected cases the fragments of the broken ulna may become more and more separated and if the skin becomes adherent a violent effort may tear open the joint 1 12. An excellent result can be obtained with or without operation, provided the olecranon does not become restricted in its movements on the humerus. The more its restriction the worse the result. Hence the mobility or immobility of the olecranon on the humerus is a very important clinical index of the value of operation in the case. 13. If the fragments are to be sutured the operation should be done as soon after the accident as the condition of the skin allows. 14. Operation is indicated in the majority of cases, particularly by wide separation of the fragments in the early period and by imperfect mobility of the olecranon in the later stages. 15. The earlier that operation is undertaken the better the prognosis of its results.

Harley-street, $\mathrm{W}$.

\section{INTESTINAL INDIGESTION.}

\section{BY P. J. CAMMIDGE, M.D, LOND.}

ARRIS AND GALE LECTURER, ROYAI COLTEGE OF SURGEONS OF ENGLAND.

DISTURBANCES of digestion are the commonest of disorders and while in a considerable number of cases they are comparatively trivial in character and quickly yield to dietetic and medicinal treatment, in others they are of a more chronic type and drag on for months or years the despair of the patients and of their medical attendants. 'Surgery may relieve some of these cases when there is a structural lesion, such as ulcer or dilatation of the stomach, underlying the condition, but in many there is no such gross anatomical change to account for the continuance of the symptoms, which are due to chemical and bacteriological abnormalities 
in the contents of the alimentary tract arising from functional changes in the digestive organs.

The work' of Pawlow, Bayliss and Starling, and others has done much to extend our knowledge of the processes involved in normal digestion and to furnish a sound basis for pathological research. The outstanding results of their investigations have been to show, first, that digestion does not consist of a series of isolated phenomena, as was at one time taught, but that each step in the process follows in an orderly manner as the result of the one which precedes it; and, secondly, that the chemical changes in the foodstuffs that fit them for absorption take place almost entirely in the upper part of the small intestine, chiefly through the agency of the various ferments contained in the secretion of the pancreas. The pathological importance of the interdependence of the various digestive processes is considerable, for if from any cause one or other of the steps is interfered with it will break the normal sequence of events and tend to bring about alterations in the chemistry, and subsequently in the flora, of the intestine that may have far-reaching results. There is, no doubt, a certain amount of compensatory activity between the various parts of the digestive system, and for a time a diminution of function in one may be more or less completely covered by the action of another, but in the end this compensation generally fails, or, as the condition becomes chronic, ceases to be adequate, and so only serves to tide over a temporary difficulty.

One of the most important links in the digestive chain is that which co-relates the functions of the stomach and pancreas. The work of the stomach is chiefly to reduce the food to such a state that it will not injure the intestine and may be readily acted on by the ferments which it is destined to meet in the succeeding parts of the alimentary tract. The function of the pancreas in digestion is to supply the main part of these ferments in such amounts, and of such kinds, as the constituents of the meal may require for their conversion into substances that can be absorbed by the intes tinal epithelium. The link connecting the activities of the two is the body called "secretin" by its discoverers, Bayliss and Starling. This is a chemical product formed by the action of the acid chyme, and to a less extent by fats and a few other substances, on the epithelial cells of the upper part of the intestine. It is absorbed by the blood and, passing to the pancreas, induces a flow of pancreatic secretion proportional in amount to the quantity of the stimulus and to the degree of acidity of the chyme. Secretin is also believed to play a part in bringing about a flow of the succus entericus, the digestive importance of which has recently been shown to be considerable.

The actual digestive changes that take place in the stomach under ordinary conditions are comparatively unimportant. Starches are not affected at all, fats are but little changed, and the proteolytic ferment of the gastric juice works very slowly, only splitting up the more readily acted on proteins to any appreciable extent. The collective pancreatic ferments are capable of acting upon all forms of foodstuff; proteins are easily and rapidly broken down into simple and readily absorbable cleavage products by the powerful tryptic ferment; starches are converted into dextrin and maltose by the amylopsin; and fats are split into their constituent fatty acids and glycerine by the steapsin. It is important to remember, however, that collagen, the chief constituent of connective tissue, is not digested by panoreatic juice unless it has been previously acted on by a dilute acid or boiled in water, so that the appearance of connective tissue in the fæces points to a disturbance of gastric digestion. The intestinal secretion, beside the "ferment of ferments," enterokinase, by which the inactive trypsinogen of the pancreatic juice is converted into active trypsin, contains erepsin, which has the power of splitting up proteoses and peptones, but not the native proteins except casein, into simpler chemical bodies and a series of ferments which convert cane-sugar, lactose, and maltose into dextrose and levulose. The succus entericus thus appears to act as a supplement of the pancreatic secretion, particularly as regards the digestion of proteins and carbohydrates.

Disturbances of the digestive functions of the pancreas and alterations in the intestinal secretion may induce abnormal changes in the food materials which will not only interfere with absorption but also give rise to the formation of unasual chemical products the presence of which may irritate the walls of the intestine. The altered reaction and composition of the intestinal contents existing under these conditions also tend to bring about a modification of the flora micro-organisms which are usually only met with in small numbers may find the conditions more suitable for their growth and so multiply to an abnormal extent; others which are normally present in large numbers in the lower bowel but are scanty in the upper part of the intestine may spread to the ileum and duodenum, and even infect the pancreatic and bile ducts, and by a process of adaptation to their environment some may develop activities which were formerly insignificant or perhaps even altogether in abeyance. Hence putrefactive decomposition may take place in the undigested, or partly digested, proteins, fats may be decomposed by fat-splitting bacteria, and carbohydrates be broken down along unusual lines into irritating organic acids, \&c., with the formation of a large amount of gas, so that the small intestine becomes the seat of changes which normally only take place to a small extent in the colon and sigmoid flexure.

The symptoms produced by such alterations in the chemistry of the digestive processes vary somewhat according to the stage of the disease and the predominance of one or other of the morbid processes. In the early stages when, as is often the case, there is hyperchlorhydria the pancreas is stimulated to unusual and abnormal activity owing to the hydrochloric acid produced in the stomach being much more than is really required for the amount and kind of food ingested. Intestinal digestion is therefore very rapid and complete, but as the pancreatic and intestinal ferments have also been poured out in excess of what is needed and, according to Starling's experiments on dogs, a mixture of pancreatic juice with the intestinal ferments gives rise to inflammatory changes and erosions in fasting animals, their presence is likely to set up some inflammatory reaction in the intestine. This is shown by abdominal discomfort, rather than pain properly speaking, which is intensified by food and becomes particularly marked when intestinal digestion is at its height, two or three hours after meals. There may be diarrhoea from the intestinal irritation but more commonly constipation is met with at first, as digestion is unusually complete. At this stage a quantitative chemical analysis of the fæces shows a low percentage of unabsorbed fat, the proportion of unsaponified fat being most markedly diminished. As the condition becomes more chronic the pancreas may fail to meet the demands upon it and undergo a certain amount of degenerative change. This may be intensified by the occurrence of an infective catarrhal pancreatitis (or "Wirsungitis"), for the catarrhal changes in the intestine give rise to conditions that are favourable to the overgrowth of bacteria and their spread upward from the lower bowel to the jejunum and duodenum, whence they may spread by direct continuity to the pancreatic ducts. There will now be added to the symptoms previously mentioned those due to imperfect digestion arising from diminution or perversion of the pancreatic secretion. The digestion of fats is, as a rule, most noticeably affected, and occasionally may be obvious on naked-eye examination of the stools. Quantitative chemical analysis of the fæces is, however, a much more reliable guide, and in most cases will show an abnormally high percentage of unabsorbed fat, with an excess of unsaponified over saponified fats. In some instances, owing to the abnormal activity of fat-splitting bacteria in the intestine, the saponified fats may be in excess. The imperfectly digested proteins present in the intestine provide a nidus for the growth of putrefactive organisms, with the result that toxic substances are formed and pass into the circulation. There are then frequently added to the symptoms of intestinal indigestion those of a toxæmia. The patient complains of constant headache, there is a feeling of weight and oppression, he is unable to concentrate his atten tion or perform any prolonged mental labour, the skin is unhealthy looking and often has a subicteric tinge, the extremities are cold, and the metatarsal joints are not uncommonly enlarged. If the liver is healthy it may for a time protect the rest of the body, but as there are of ten an infection of the biliary passages and some interference with the functions of the liver toxæmia is a common complication. Examination of the stools may show a number of undigested and partly digested muscle fibres, but if the functions of the stomach are being satisfactorily carried out 
these will be found as individual fibres and not as associated bundles. Carbohydrates, instead of being digested and absorbed in the usual way, are apt to be broken down by the action of the intestinal bacteria into organic acids and gases, so that abdominal distension, gaseous eructations, and the passage of flatus occur.

In some instances the disease appears to be due to a bacterial infection from the commencement and may occasionally be traced to an antecedent attack of influenza or typhoid fever. In such cases there are nearly always an associated pancreatitis and cholangitis and it is to the former that the disturbances of digestion are chiefly due. The attacks in these cases are often of a remittent character but, although the patient may be comparatively well in the intervals, he is never quite free from discomfort. Certain articles of diet and particular forms of food material are frequently found to increase the discomfort and to precipitate an attack; fatty foods, and particularly some forms of fat, are often digested with difficulty and several patients have voluntarily informed me that milk was "poison" to them.

The diagnosis of intestinal indigestion is made partly from the clinical symptoms and partly from the results of an analysis of the fæces, urine, and stomach contents. Each supplies a certain amount of information but no one affords a pathognomonic sign. A correct conception of the conditions present in any particular case can only be arrived at by taking into consideration the information to be obtained from them all. The presence of abdominal discomfort, more marked two or three hours after food, loss of appetite and distaste for certain articles of diet-fat or milk, for example -lassitude, inability to concentrate the attention, with often persistent headache, a dirty tongue which is frequently fissured, foetid breath with eructations of gas, abdominal distension, and cccasional tenderness on deep pressure in the region of the head of the pancreas, the passage of much flatus with often foul-smelling stools, and either diarrhoea or constipation are suggestive of the condition.

The analysis of the fæces should be as complete and exhaustive as possible, for the macroscopical, microscopical, chemical, and frequently the bacteriological characters all assist in arriving at a conclusion as to the nature and extent of the disease. Although much may be learnt from an investigation of the fæces passed on an ordinary mixed diet it is advisable in some instances to place the patient on a test diet, such as that suggested by Schmidt, ${ }^{1}$ when a more accurate estimate of the digestive disturbances can be made. Information as to the particular kinds of food-stuffs that are undigested may also be obtained by the "bead-test," in which pieces of raw catgut, meat, potato, and mutton fat are attached to coloured beads and given by the mouth in a capsule, the beads being afterwards recovered from the fæces and examined.

In the analysis of the urine attention must be particularly devoted to the "pancreatic " reaction, " as indicating active degenerative changes in the pancreas and involvement of the gland, the presence of an excess of indican and a disturbed relation between the preformed and ethereal sulphates, as showing abnormal putrefactive changes in the intestinal contents with catarrh of the walls, and a marked reaction for urobilin, which in my experience points to an associated cholangitis and disturbance of the liver.

The stomach contents should be examined both before and after a test meal for evidences of hyperchlorhydria, catarrh, dilatation, \&c., as the intestinal condition is often associated with, and secondary to, disease of the stomach. To obtain really useful information it is necessary that the meal should be of a definite and simple character, and that the test should be carried out under certain conditions. The most generally useful test meal is the Ewald-Boas breakfast, consisting of 35 grammes (about two slices) of bread, or toast, without butter, and 400 cubic centimetres (about two cups) of water or weak tea without milk or sugar. This should be withdrawn not less than three-quarters of an hour and not more than one hour after it has been taken. In regaining it no water should be used as this would vitiate the quantitative analysis and so deprive the test of the greater part of its value; if the patient is unable to return the meal through the tube spontaneously some simple form of suction apparatus, such as that of Senorans, should be used.

1 The Test-Diet in Intestinal Diseases, Adolf Schmidt, translated by C. D. Aaron (Davis and Co., Philadelphia, 1896)

C. D. Aaron (Davis and Co., Philadelphia, 1896). 2 The Pancreas, its Surgery and Patl
Cammidge, p. 252 (Saunders and Co., 1908).
The treatment is based upon the pathological findings and will vary with the cause of the condition and the degree to which the various factors mentioned contribute to the resnlt. The choice of a suitable diet is the first consideration. The guide to this is the result of the examination of the fæces, from which may be learnt the types of food that are undigested and are contributing most to the irritation of the intestine. The meals should be arranged so that there is the longest possible interval between them in order that the digestive organs may be given a maximum amount of physiological rest, and if one meal is made as far as may be carbohydrate, while the next is chiefly protein, this end is contributed to, Foods rich in proteins are to be recommended in cases where the production of hydrochloric acid is found to be excessive, and in these cases, too, an eggspoonful of a mixture of equal parts of sodium bicarbonate, magnesium carbonate, and calcium carbonate after meals, or 1 to 3 parts of hydrogen dioxide in 200 to 300 cubic centimetres of water in the morning, fasting, as suggested by Petri, ${ }^{3}$ are also useful in controlling the hyperchlorhydria.

In cases where the bacterial element predominates and there is evidence of toxæmia the use of butter-milk, or, better, of a preparation of milk "soured" with a reliable culture of the Bulgarian lactic acid bacillus, often gives very good results. In some cases, however, the milk has appeared rather to aggravate the intestinal discomfort and has had to be given up. In several instances I have been able to relieve the toxic symptoms by inoculations, or the administration by the mouth on an empty stomach, of vaccines prepared from cultures of the predominant organisms in the fæces. Castor oil or calomel in doses of from one to four grains is a useful preliminary to other treatment, and I always advise that a blue pill and black draught should be taken at the commencement of the treatment and once a month afterwards, even after the symptoms have subsided.

Where the results of the "pancreatic" reaction in the urine and the analysis of the fæces point to involvement of the pancreas the cause of the pancreatitis must first be determined. Pancreatitis is never a primary disease, although the symptoms to which it gives rise may be the dominant features of the case, and to attempt the cure of the pancreatic condition without at the same time treating the cause is to labour in vain. If infection of the duodenum and pancreatic ducts is present the treatment of the former should be undertaken along the lines already indicated. The most useful drug for dealing with the infection of the pancreatic duct and biliary passages is undoubtedly urotropine. ${ }^{4}$ I have seen cases that have cleared up in a week or ten days after commencing to use it. As it is somewhat irritating and may therefore possibly increase the inflammatory reaction in the pancreas when given in large doses, I usually prescribe 5 grains, well diluted, three times a day. Salicylates, too, are of use in these cases as they also are excreted by the pancreas and exert an antiseptic action on the contents of the ducts, but they do not appear to act as quickly or efficiently as urotropine.

In the more chronic cases, with marked disturbance of the functions of the pancreas, medicinal treatment may prove of no avail and resort must then be had to surgical measures, the infected ducts being drained through the common duct and gall-bladder by the operation of cholecystotomy or cholecyst-enterostomy.

The early recognition and treatment of intestinal indigestion are primarily important because of the discomfort the condition canses the patient and also because of the interference with nutrition that it brings about, but the complications and sequelæ that are liable to occur in chronic cases are of no less moment. Toxæmia may entirely unfit the patient for any occupation involving mental strain or concentration, and one finds that a considerable number drift into that somewhat heterogeneous group of cases provisionally labelled "neurasthenia." A remote consequence of chronic intestinal indigestion, but one which I am convinced is not to be despised, is the ultimate occurrence of diabetes. This is apparently due to involvement of the pancreas in the disease, and although we do not at present know for certain why some cases of chronic pancreatitis due to infection of the ducts should develop glycosuria, while other, and apparently quite similar cases, do not, the fact remains that it is not a negligible possibility. The progress of the disease is

3 Archiv für Verdauungs-Krankheiten, Band xiv., p. 479

4 S. J. Crowe: Johns Hopkins Hospital Bulletin, Apri, 1908, p. 109. 
usually slow and although I have notes of several cases in which glycosuria has occurred within a year or two of the first appearance of the symptoms of intestinal indigestion others have dated back the initial attack ten or 15 years. I have for some time maintained that the treatment of diabetes is to a large extent prophylactic, ${ }^{5}$ and $I$ believe that in the early treatment of disturbances of the chemistry and bacteriology of the upper intestine lies, at any rate in part, the solution of a difficult problem.

Beaumont-street, $\mathrm{w}$.

\section{ON THE CAUSE OF ACHROMASTA IN LEUCOCYTES. ${ }^{1}$}

BY H. C. ROSS, L.R.C.P. LoND., M.R.C.S. ENG., IATE"SURGEON, ROYAL NAVY; PATHOLOGIST TO THE ROYAL SOLTHERN HOSPITAL, LIVERPOOL.

IN recent publications $(1,2,3) *$ I have drawn attention to a method of examining living blood cells by spreading them upon agar jelly which contains stain and salts in solution. These papers have described how the leucocytes absorb the stain during life, and that their granules and cytoplasm become coloured, but that the colouration does not apparently affect their life since amoboid movements continue. However, as soon as their nuclei stain, death ensued, and liquefaction of the cytoplasm then occurs, followed by a gradual, and in certain circumstances rapid, loss of stain. This loss of stain being a remarkable phenomenon required further investigation. The present paper describes the result of this investigation with an enumeration of a series of associated events which occur in cells shortly after death, and I shall endeavour to show the cause of the loss of stain by connecting these events together.

The researches already referred to have demonstrated that the cytoplasm of leucocytes appears to present jelly-like properties and that the diffusion of stain into it is hastened by heat and alkalies and is delayed by neutral salts. Consequently, when the cells are resting on agar which being of a certain temperature contains also a sufficiency of stain and alkali to stain the nuclei, death is occasioned, and it occurs either just before or just after the staining of the nucleus, for I am unable to determine whether the staining of the nucleus causes death, or whether death allows the nucleus to stain. The fact remains, however, that staining of the cytoplasm and granules does not appear to be very detrimental, for leucocytes will even show excited movements under these conditions, but with a stained nucleus they are invariably immobile, even on a jelly which contains stimulating constituents (3).

If the stained agar film on which the cells are resting contains sufficient alkali and heat to cause the stain to diffuse into the cells to such an extent as to produce staining of the nucleus and death, the cytoplasm begins to liquefy as demonstrated by the onset of Brownian movement of the granules, and it has already been shown that liquefaction of the cytoplasm begins at the periphery and progresses towards the nucleus (2). Then a remarkable thing happens. The nucleus gradually loses its stain until it appears as unstained as it was when the cell was placed on the agar.

The researches also showed that this loss of stain from stained leucocytes occurs with varying rapidities. For instance, in Experiment 1, given in the paper "On the Death of Leucocytes" (1), when the agar on which the cells are resting only contained stain and no salts "the nuclei and granules of these cells remained stained for two or three hours; when, however, they burst, their stained nuclei and granules were discharged and remained stained a purple colour, so that ultimately the slide contained many free and stained nuclei floating about under the cover-glass." In . Experiment 3, however, in the same paper, when the agar contained the salts, sodium citrate and sodium chloride, the following occurred: "In about half an hour after staining of the nucleus the stained part of the cytoplasm gave up its

\footnotetext{
5 Pancreatic Diabetes (Surgery, Gynecology, and Obstetrics, January, 1908, p. 22).

1 The word leucocyte in this paper refers to the polymorphonuclear leucocyte.

* The figures within parentheses refer to the bibliography at the end of the article.
}

stain, and then the nucleus and granules did the same until the whole cell was as unstained as it appeared when the slide was prepared." Both of these experiments were conducted at a temperature of $30^{\circ} \mathrm{C}$. I will endeavour to show that there is an intimate relationship between this loss of tain from the nucleus and the liquefaction of the cytoplasm.

Definition. - A leucocyte may be defined as being achromatic when its stained nucleus has lost its stain, or when its unstained nucleus refuses to stain with the cell resting in a medium which will stain the nuclei of fresh normal lencocytes.

Methods adopted for demonstrating achromasia.-1. Some blood was citrated and kept at a temperature of $30^{\circ} \mathrm{C}$. for four days, when it was found that many of the nuclei of the leucocytes refused to stain by the ordinary methods of staining blood.

2. Blood was examined at a temperature of $30^{\circ} \mathrm{C}$. on a film prepared from a jelly, 6 cubic centimetres of which contained 1 cubic centimetre of Unna's polychrome methylene blue (Grubler) and 5 cubic centimetres of water. It also contained in solution 1 per cent. of agar, 1 per cent. of sodium citrate, and 0.5 per cent. of sodium chloride, and it was rendered alkaline by the addition of 0.4 cubic centimetre of a 5 per cent. solution of sodium bicarbonate ${ }^{2}$ The nuclei stained deeply in ten minutes and then slowly lost their stain. After the loss another part of the same agar film stained the nuclei of fresh normal leucocytes.

3 . 2 per cent. of sodium citrate and 0.5 per cent. of sodium chloride were added to some Unna's stain. A volume was mixed with an equal volume of blood and kept at $37^{\circ} \mathrm{C}$. for 15 minutes. An agar film was prepared as in Method 2. The corpuscles in the incubated mixture were examined on this film and were seen to be deeply stained. The slide was then incubated for ten minutes, it having previously been shown that at this temperature there was sufficient concentration of stain and alkali in the agar to stain fresh normal leucocytes in ten minutes. It was found, however, on removal from the incubator that the stained nuclei had lost their stain, though many of the cells were intact and not ruptured.

Since cells kept for four days are dead (3), and since staining of the nuclei is a sign of death (1), I conclude that acbromasia is due to a change taking place in the cells after death, for it is evident that the stain remains unchanged as it will stain the nuclei of fresh normal leucocytes.

Effect of heat on achromasia.-.-Method 2 was repeated, but with a temperature of $20^{\circ}$ instead of $30^{\circ} \mathrm{C} .0 .5$ cubic centimetre instead of 0.4 cubic centimetre of alkali solution bad to be added to produce staining in the same time $(1,3)$ Few of the nuclei lost their stain until rupture of the cells. I hence conclude that heat accelerates achromasia.

Effeots of salts and rupture on achromasia. - Method 2 was repeated and the experiment given in the last paragraph was also repeated; at either temperature $\left(20^{\circ} \mathrm{C}\right.$. or $30^{\circ} \mathrm{C}$.) if a cell ruptured it lost its stain immediately. But in the first experiment quoted from a former paper, when salts were absent, it was stated that when the cells ruptured, free, stained nuclei were to be seen floating under the cover-glass. I conclude that rupture causes immediate achromasia in the presence of salts but not in the absence of salts.

Effect of heat and salts on liquefied oytoplasm.-Previously to incubation of the slide for ten minutes, as described in Method 3, the granules were showing Brownian movement whereas after the incubation the granules appeared quite fixed, and since Brownian movement of the granules is a sign of liquefaction of the cytoplasm (1), it is manifest that the incubation for ten minutes had in some way affected the cell contents. Yet in the first experiment in the previous paper, part of which has already been quoted, it was shown that Brownian movement continued for a long time on agar which contained no salts although the slide be kept at $30^{\circ} \mathrm{C}$. In this last experiment, therefore, heat does not apparently affect liquefied cytoplasm. The inference is that liquefied cytoplasm will pass out of the cells by osmosis in the presence of heat and salts, in which case the cell collapses and the granules appear fixed; but if no salts are present osmosis will not occur even at a comparatively high temperature.

Experiments to show the relationship betreen osmosis of the cytuplasm and the onset of uchromasia. When Method 3 was repeated but without incubation of the slide achromasia did not take place for a long time at a temperature of $15^{\circ} \mathrm{O}$., 